TRABAJOS ORIGINALES

Rev Obstet Ginecol Venez. 2020; 80 (4): 292-302

https://doi.org/10.51288/00800406

\title{
Listas de chequeo en obstetricia: ayudas cognitivas que salvan vidas
}

\author{
Drs. Juan Pérez Wulff, ${ }^{1}$ Daniel Márquez C, ${ }^{2}$ Jesús Veróes, ${ }^{3}$ Jonel Di Muro, ${ }^{3}$ Carlos Lugo, ${ }^{3}$ \\ Rafael Cortés, ${ }^{4}$ Susana De Vita, ${ }^{3}$ Kenny Araujo, ${ }^{3}$ Edinson Valencia, ${ }^{5}$ Stefanía Robles ${ }^{5}$.
}

\begin{abstract}
RESUMEN
Objetivo: Proponer la utilización de ocho listas de chequeo en las salas de emergencia obstétrica del país.

Métodos: Se constituyó un equipo interdisciplinario con médicos especialistas en obstetricia y ginecología, perinatología, medicina materno fetal, medicina crítica, anestesiología, infectología y neonatología. Previa determinación de las principales patologías que inciden en la mortalidad materna en Venezuela, se revisaron múltiples listas de chequeo utilizadas en diferentes centros a nivel mundial, publicaciones disponibles en bases de datos y opiniones de expertos. Se adecuaron a las realidades del país y la disponibilidad médica y se priorizaron las acciones médicas, pruebas de laboratorio, tratamiento farmacológico y elementos diagnósticos.

Resultados: Se presentan las listas de chequeo para hemorragia posparto, sepsis obstétrica, trastornos hipertensivos del embarazo (preeclampsia con signos de gravedad y eclampsia), intoxicación por sulfato de magnesio, acretismo placentario, resucitación cardiopulmonar materna y trauma y embarazo.

Conclusión: Las listas de chequeo producto de la iniciativa de la Sociedad de Obstetricia y Ginecología de Venezuela quedan a disposición de todo el personal de salud que las requiera para su implementación en escenarios educativos de simulación y en el ejercicio clínico, como una herramienta adicional para la búsqueda de mejores desenlaces en los pacientes que requieren un manejo de alta complejidad en salas de maternidad.
\end{abstract}

Palabras clave: Lista de chequeo, Hemorragia posparto, Sepsis obstétrica, Trastornos hipertensivos del embarazo, Intoxicación por sulfato de magnesio, Acretismo placentario, Resucitación cardiopulmonar materna, Trauma y embarazo.

\section{SUMMARY}

Objective: To propose the use of eight checklists in the country's obstetric emergency rooms.

Methods: An interdisciplinary team was established with physicians specializing in obstetrics and gynecology, perinatology, fetal maternal medicine, critical medicine, anesthesiology, infectology and neonatology. Upon determination of the main pathologies affecting maternal mortality in Venezuela, multiple checklists used in different centers worldwide, publications available in databases and expert opinions were reviewed. They adapted to the realities of the country and medical availability and prioritized medical actions, laboratory tests, drug treatment and diagnostic elements.

Results: Checklists for postpartum hemorrhage, obstetric sepsis, hypertensive pregnancy disorders (preeclampsia with signs of severity and eclampsia), magnesium sulfate poisoning, placental acretism, maternal cardiopulmonary resuscitation, and trauma and pregnancy are presented.

Conclusion: The checklists resulting from the initiative of the Society of Obstetrics and Gynecology of Venezuela are available to all health personnel who require them for implementation in educational simulation scenarios and in clinical practice, as an additional tool for finding better outcomes in patients who require high complexity management in maternity rooms.

Keywords: Checklist, Postpartum hemorrhage, Obstetric sepsis, Hypertensive pregnancy disorders, Magnesium sulfate poisoning, Placental acretism, Maternal cardiopulmonary resuscitation, Trauma and pregnancy

${ }^{1}$ Especialista en Perinatología y Medicina Materno-Fetal, Director Advanced Life Support in Obstetrics (ALSO), Coordinador regional del comité de medicina materno fetal Federación Latinoamericana de Sociedades de Obstetricia y Ginecología (FLASOG). ${ }^{2}$ Especialista en Perinatología y Medicina Materno-Fetal, Especialista en Medicina Crítica, Instructor Advanced Life Support in Obstetrics (ALSO), Coordinador regional del comité de medicina materno fetal Federación Latinoamericana de Sociedades de Obstetricia y Ginecología (FLASOG). ${ }^{3}$ Especialista en Perinatología y Medicina Materno-Fetal, Instructor Advanced Life Support in Obstetrics (ALSO). ${ }^{4}$ Especialista en Perinatología y Medicina MaternoFetal, Director Advanced Life Support in Obstetrics (ALSO). Coordinador regional del comité de medicina materno fetal Federación latinoamericana de Sociedades de Obstetricia y Ginecología (FLASOG). ${ }^{5}$ Especialista en Perinatología y Medicina Materno-Fetal

\section{INTRODUCCIÓN}

En Venezuela, las tres principales causas de muerte materna son hemorragia posparto (HPP), trastornos hipertensivos del embarazo (THE) y sepsis (1). El trauma representa la primera causa de morbimortalidad no obstétrica en embarazadas (2). Estos eventos críticos constituyen situaciones estresantes y potencialmente fatales que requieren un manejo oportuno, rápido y 
coordinado para obtener un desenlace exitoso. Son emergencias en las que el desempeño del equipo de salud puede ser un determinante en la probabilidad de sobrevida de las pacientes. La incapacidad para manejar adecuadamente las situaciones que amenazan la vida ha sido reconocida como la mayor fuente de variación en la mortalidad intraoperatoria $(3,4)$. En el caso de la paciente obstétrica, esta situación se agrava al tratarse de situaciones de alto riesgo para el binomio madre-feto (5).

Las emergencias en obstetricia representan un verdadero desafío para el personal de salud. La capacidad de respuesta no depende solamente de habilidades técnicas, sino también de competencias no técnicas (6). Estudios previos (7) han destacado que las herramientas de desarrollo interpersonal, sociales y cognitivas juegan un papel importante en la respuesta de los médicos ante la crisis. Entre las competencias no técnicas se incluye, además del conocimiento, la actitud, la colaboración, el estado de alerta y la capacidad de liderazgo. Estos elementos son indispensables para la obtención de resultados perinatales satisfactorios.

Las ayudas cognitivas son asistentes para la memoria que contienen información importante presentada de manera análoga o digital, que recuerdan instrucciones diagnósticas y correctivas para el manejo de situaciones especiales (8). Son herramientas que apoyan la toma de decisiones y no solo están diseñadas para personal en entrenamiento, pues no son simplemente ayudas para el aprendizaje (9). Las ayudas cognitivas se pueden presentar como algoritmos, mnemotecnias y listas de chequeo o verificación, entre otras (10). Su uso ha mejorado los resultados en una gran variedad de escenarios clínicos, siendo una herramienta sencilla y de bajo costo, fácil de aplicar, reproducible, válida y fiable (11). Adicionalmente, su puesta en práctica junto a competencias no técnicas de comunicación (12) promueve una interacción interdisciplinaria adecuada durante la crisis y por tanto, una mejoría en el desempeño del equipo que afronta la situación de emergencia $(13,14)$.

Todo el equipo médico y paramédico debe tener conocimiento del manejo adecuado de la patología a tratar, así como la necesidad de recibir entrenamiento continuo y actualizado en el abordaje de la emergencia. El uso de listas de chequeo en la atención de eventos críticos se ha correlacionado con un mejoramiento en la adherencia a las guías clínicas (15). La evidencia sugiere que las listas de chequeo tienen un efecto favorable sobre la coordinación, comunicación y desempeño general de los equipos clínicos de trabajo, y que su diseño lineal podría tener ventajas comparado con el diseño ramificado de los algoritmos $(10,16)$.

Es por ello que se propone la utilización de ocho listas de chequeo en las salas de emergencia obstétrica, desarrolladas y enfocadas en el manejo sistemático, ordenado y preciso de las patologías que ocupan las principales causas de mortalidad materna en Venezuela, facilitando de esta manera su uso por parte del equipo médico de choque (disponibles en: www. sogv.com.ve).

\section{MÉTODOS}

Se procedió de la siguiente manera para la elaboración de las listas de chequeo:

a) Constitución de un equipo interdisciplinario en el que participaron médicos especialistas en obstetricia y ginecología, perinatología, medicina materno fetal, medicina crítica, anestesiología, infectología y neonatología.

b) Determinación de las principales patologías que inciden en la mortalidad materna en Venezuela.

c) Revisión de múltiples listas de chequeo utilizadas en diferentes centros a nivel mundial, 
publicaciones disponibles en bases de datos y opiniones de expertos $(7-13,17)$.

d) Adecuación de las listas de chequeo a las realidades del país y la disponibilidad médica.

e) Priorización de las acciones médicas, pruebas de laboratorio, tratamiento farmacológico y elementos diagnósticos.

\section{RESULTADOS}

Lista de chequeo número 1. Hemorragia posparto

La hemorragia posparto continúa siendo la primera de causa de muerte materna a nivel mundial. Está ampliamente demostrado que los cambios fisiológicos maternos producen variaciones en los parámetros hemodinámicos, que pueden enmascarar la pérdida de sangre en etapas iniciales, pero que provocan una rápida descompensación en la gestante (18). Resulta indispensable el entrenamiento del personal médico y paramédico en el reconocimiento temprano de los signos de alarma y la instauración rápida de medidas destinadas al control del sangrado y soporte de reanimación básica en la paciente obstétrica complicada con HPP.

Lista de chequeo número 2. Sepsis obstétrica.

La sepsis obstétrica se asocia a una importante morbimortalidad materna. $\mathrm{Su}$ reconocimiento, diagnóstico temprano y manejo oportuno pueden contribuir a disminuir las altas cifras de mortalidad por causa infecciosa (19). Es importante recordar que los cambios fisiológicos en la embarazada pueden enmascarar los signos precoces de infección, además

Lista de chequeo número 1 . Hemorragia posparto

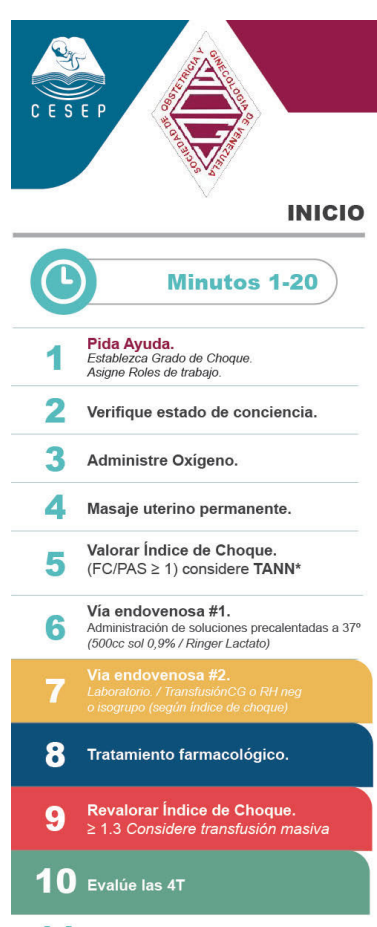

11 Temperatura corporal y diuresis.

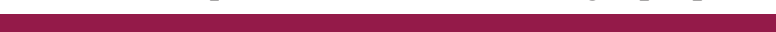

Hemorragia Posparto

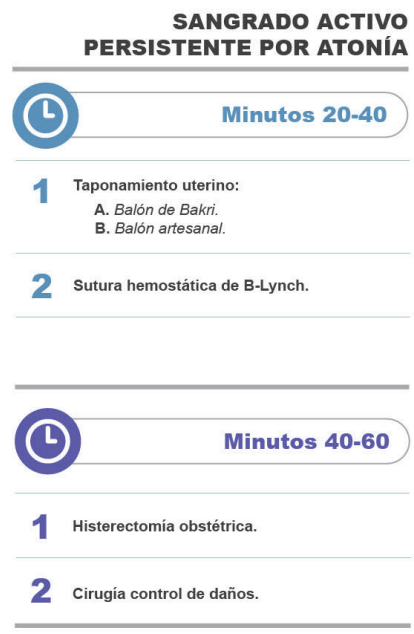

* TANN: Traje antichoque no neumático

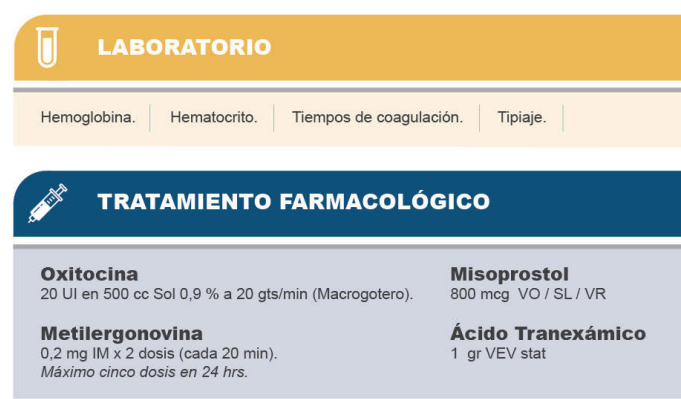

TRANSFUSIÓN MASIVA

6 CG - 6 PFC - 6 CP

determine LAS 4T

\begin{tabular}{ll}
\hline $\begin{array}{l}\text { Tono: } \mathbf{7 0} \% \\
\text { Masaje uterino y fármacos. }\end{array}$ & $\begin{array}{l}\text { Tejido: } \mathbf{9} \% \\
\text { Remoción de restos. }\end{array}$ \\
$\begin{array}{l}\text { Trauma: } \mathbf{2 0} \% \\
\text { Sutura de desgarros. }\end{array}$ & $\begin{array}{l}\text { Trombina: } \mathbf{1} \% \\
\text { PFC y /o crioprecipitado. }\end{array}$ \\
\hline
\end{tabular}


Lista de chequeo número 2. Sepsis obstétrica.

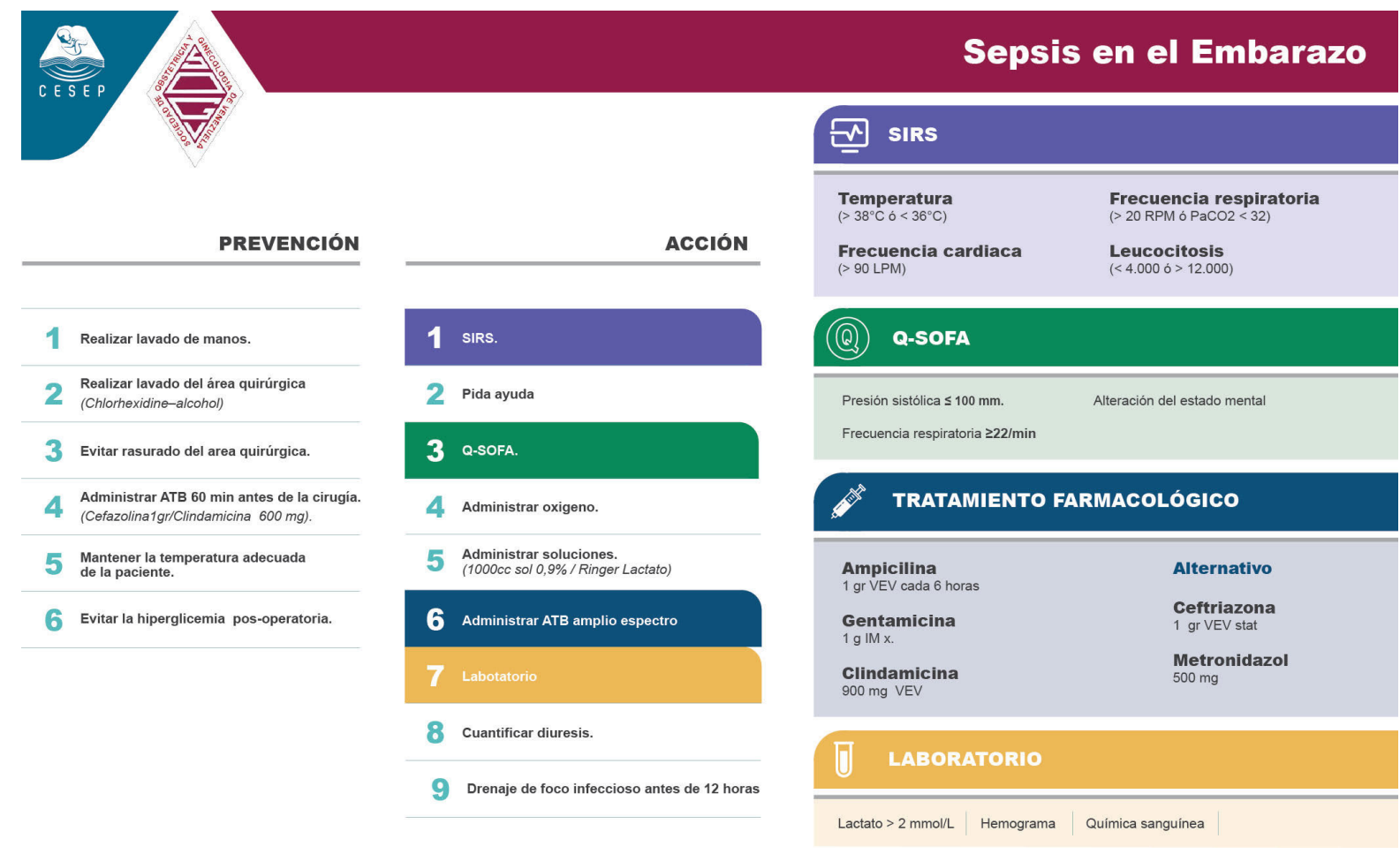

de aumentar la susceptibilidad de padecer infecciones. La fiebre constituye un importante marcador de riesgo infeccioso. La aplicación de paquetes de intervención, bajo listas de chequeo, ha mostrado ser una medida efectiva en la reducción del choque séptico obstétrico (20).

Lista de chequeo número 3. Trastornos hipertensivos del embarazo. Preeclampsia con signos de gravedad.

Los trastornos hipertensivos durante la gestación constituyen una de las tres principales causas de mortalidad materna y se estima que complica del $2 \%$ al $8 \%$ de los embarazos a nivel mundial. En América Latina y el Caribe contribuyen con el $26 \%$ de la mortalidad materna (21). Todo el personal de salud debe conocer los criterios diagnósticos, alteraciones de laboratorio y parámetros de gravedad asociados a los THE, disminuyendo así el tiempo de atención y la morbimortalidad perinatal asociada.

Lista de chequeo número 4. Trastornos hipertensivos del embarazo. Eclampsia.

La eclampsia constituye la manifestación convulsiva de la hipertensión durante la gestación. Representa una causa importante de mortalidad materna, principalmente en países en vías de desarrollo (22, 23). Las convulsiones tónico-clónicas precedidas por cefalea, visión borrosa, escotomas e hiperreflexia, pueden ocurrir antes, durante o después del nacimiento. El rápido y adecuado manejo mediante listas de chequeo durante la emergencia mejorará los resultados materno-fetales. 
Lista de chequeo número 3. Trastornos hipertensivos del embarazo. Preeclampsia con signos de gravedad.

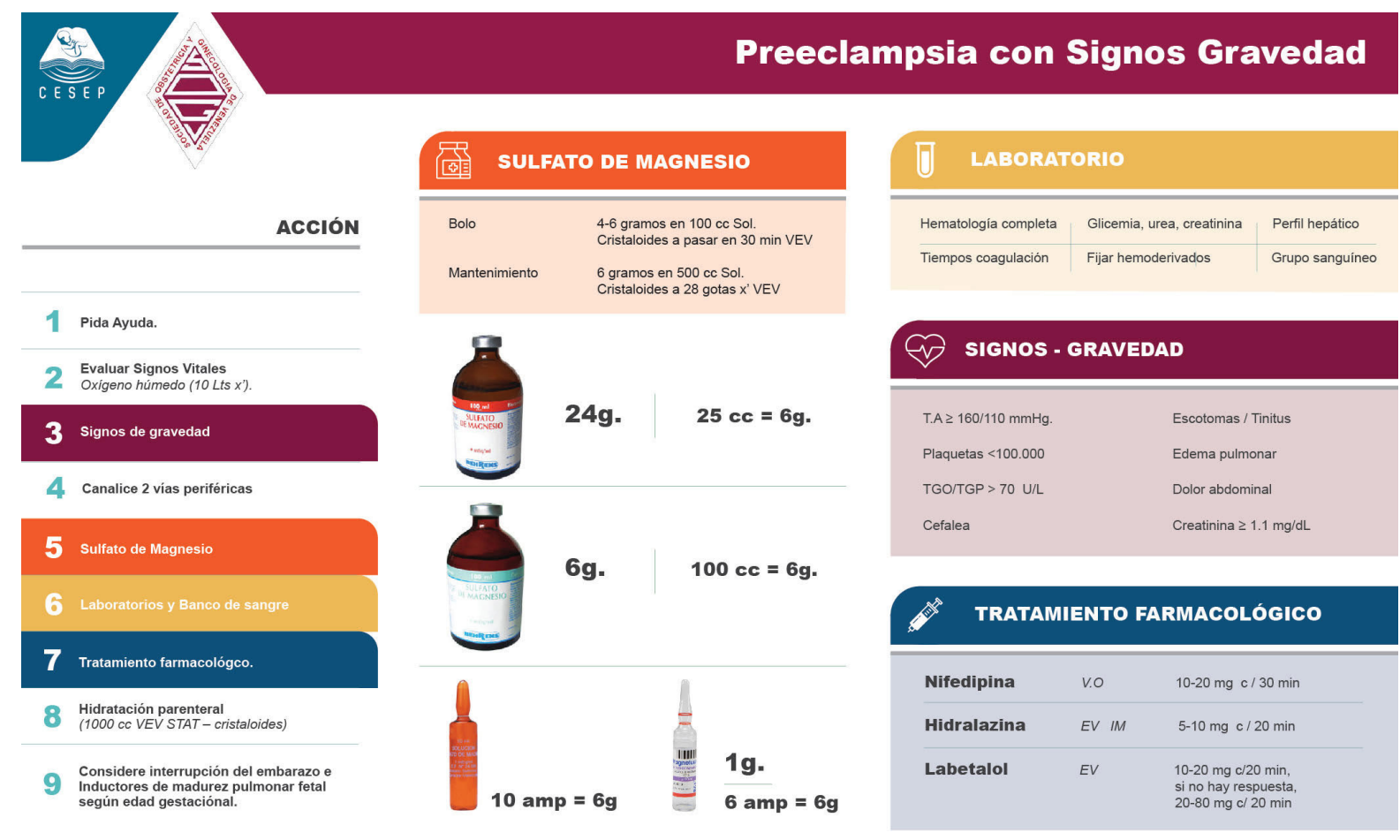

Lista de chequeo número 4. Trastornos hipertensivos del embarazo. Eclampsia.

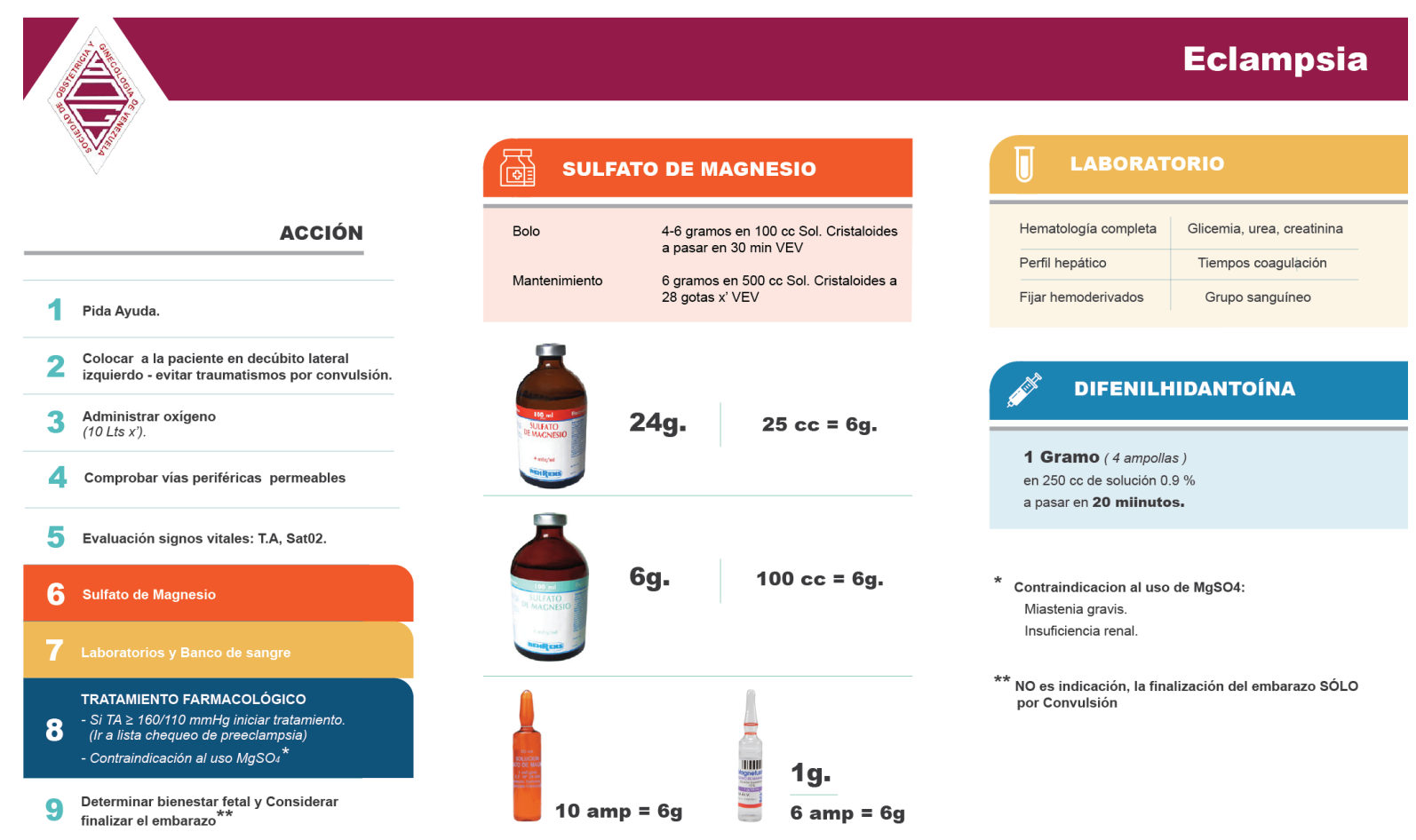


Lista de chequeo número 5. Intoxicación por sulfato de magnesio

El sulfato de magnesio es considerado el medicamento de elección durante las convulsiones asociadas a trastornos hipertensivos, a pesar de ello, la posibilidad de intoxicación por sulfato de magnesio en las salas de emergencia es frecuente. (21-23) Las listas de chequeo permiten el manejo sistemático y adecuado frente a la intoxicación por sulfato de magnesio, reduciendo el tiempo de manejo y previniendo complicaciones potencialmente mortales.

Lista de chequeo número 6 . Acretismo placentario.

Dentro de la hemorragia posparto, el acretismo placentario contribuye en gran medida con la tasa de mortalidad materna, especialmente cuando no se ha realizado el diagnóstico antenatal. La prevalencia del espectro placenta ácreta se ha incrementado de manera exponencial debido al uso y abuso de la operación cesárea. Con cada nueva cirugía obstétrica hay un aumento considerable de riesgo para las anomalías de inserción placentaria. Actualmente, la combinación de placenta previa y antecedente de cesárea conlleva a plantear de entrada la probabilidad de un acretismo placentario (24-26). El siguiente protocolo de atención institucional contempla el uso de la palabra ACRETISMO como acrónimo, simplificando la toma de decisiones y favoreciendo conductas sistemáticas para el manejo de esta entidad.

Lista de chequeo número 7. Resucitación cardiopulmonar materna.

A nivel mundial, el paro cardíaco ocurre en

Lista de chequeo número 5. Intoxicación por sulfato de magnesio.

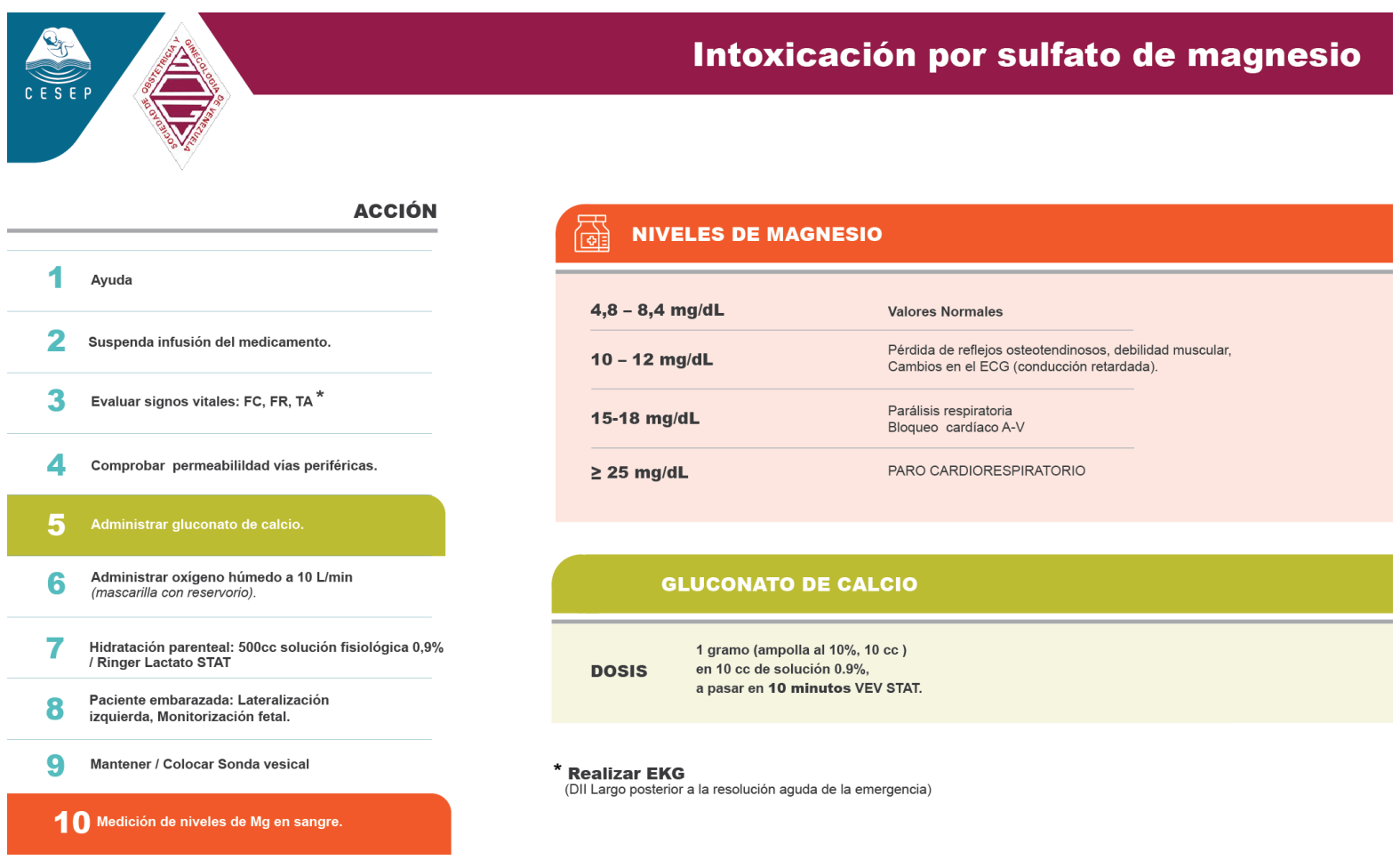

Vol. 80, $\mathrm{N}^{\mathrm{0}}$ 4, diciembre 2020. 
Lista de chequeo número 6. Acretismo placentario.

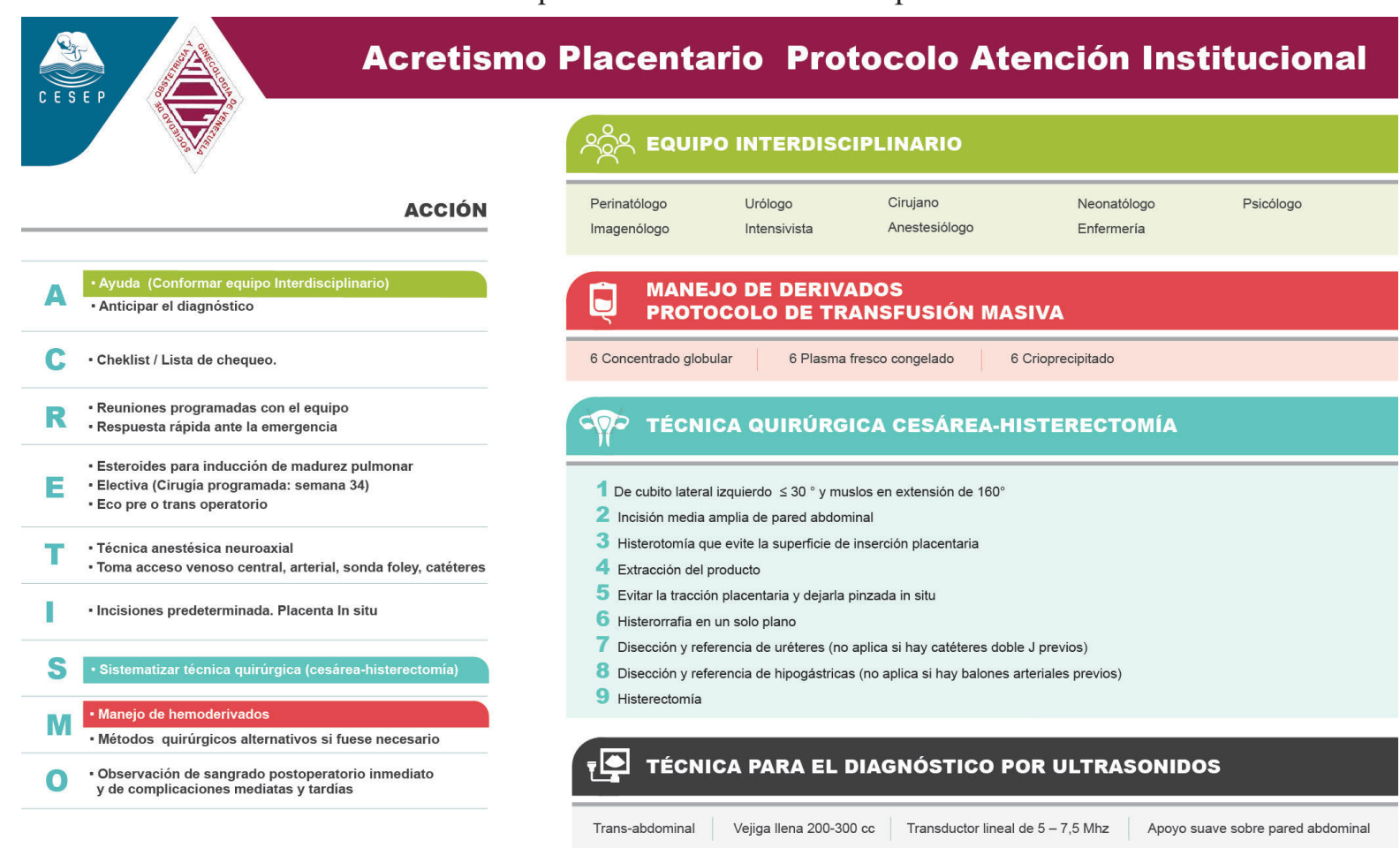

Lista de chequeo número 7. Resucitación cardiopulmonar materna.

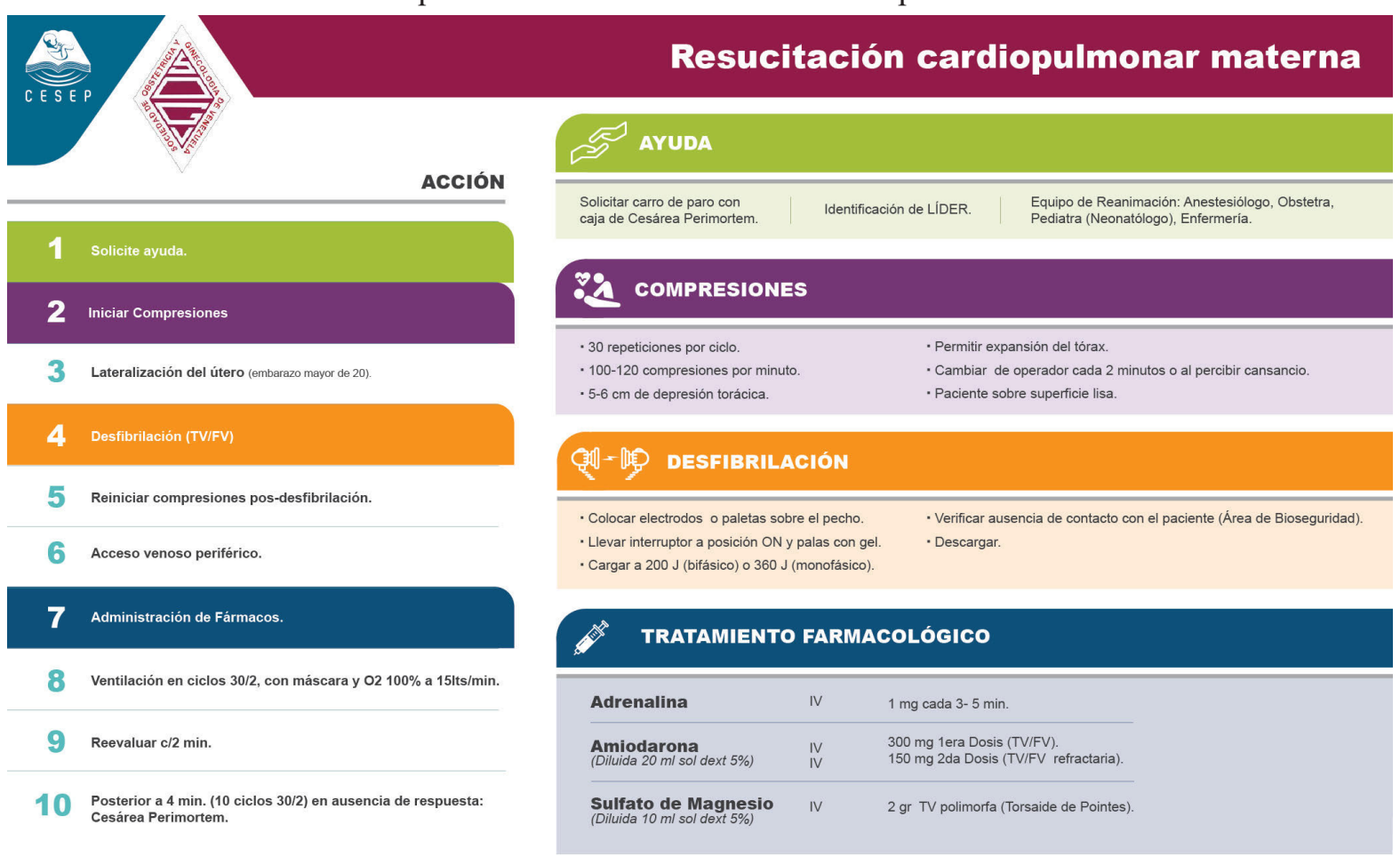


1:12 000 admisiones obstétricas (27). Aunque la técnica de reanimación en la embarazada es similar a la de población no obstétrica, varios aspectos y consideraciones son excepcionalmente diferentes debido a los cambios fisiológicos del embarazo. Es necesaria la lateralización del útero hacia el lado izquierdo para mejorar el retorno de sangre hacia el corazón. Del mismo modo resulta de particular relevancia la semana 20 de gestación, punto corte para la realización de la histerotomía (cesárea) perimortem. El enfoque actual de la reanimación cardiopulmonar en embarazadas durante el tercer trimestre parte de la adherencia a la "regla de los cuatro minutos" (28): si los pulsos no han regresado dentro de los 4 minutos posteriores al inicio de la reanimación, debe practicarse la cesárea perimortem, dados los beneficios hemodinámicos de la descompresión uterina y la mejora del retorno venoso (29). El algoritmo representa una guía para la atención del colapso materno, basado en aprendizaje por simulación y aplicable al contexto clínico.

Lista de chequeo número 8 . Trauma y embarazo

Las listas de chequeo o verificación han tenido un impacto importante en los escenarios anteriores y surgen como parte fundamental del abordaje del paciente politraumatizado (30). El manejo de la embarazada con trauma ha sido objeto de múltiples reportes (31). En Venezuela hay un creciente aumento de cifras relacionadas con estos eventos por lo que es importante considerar el siguiente protocolo de manejo.

Lista de chequeo número 8 . Trauma y embarazo

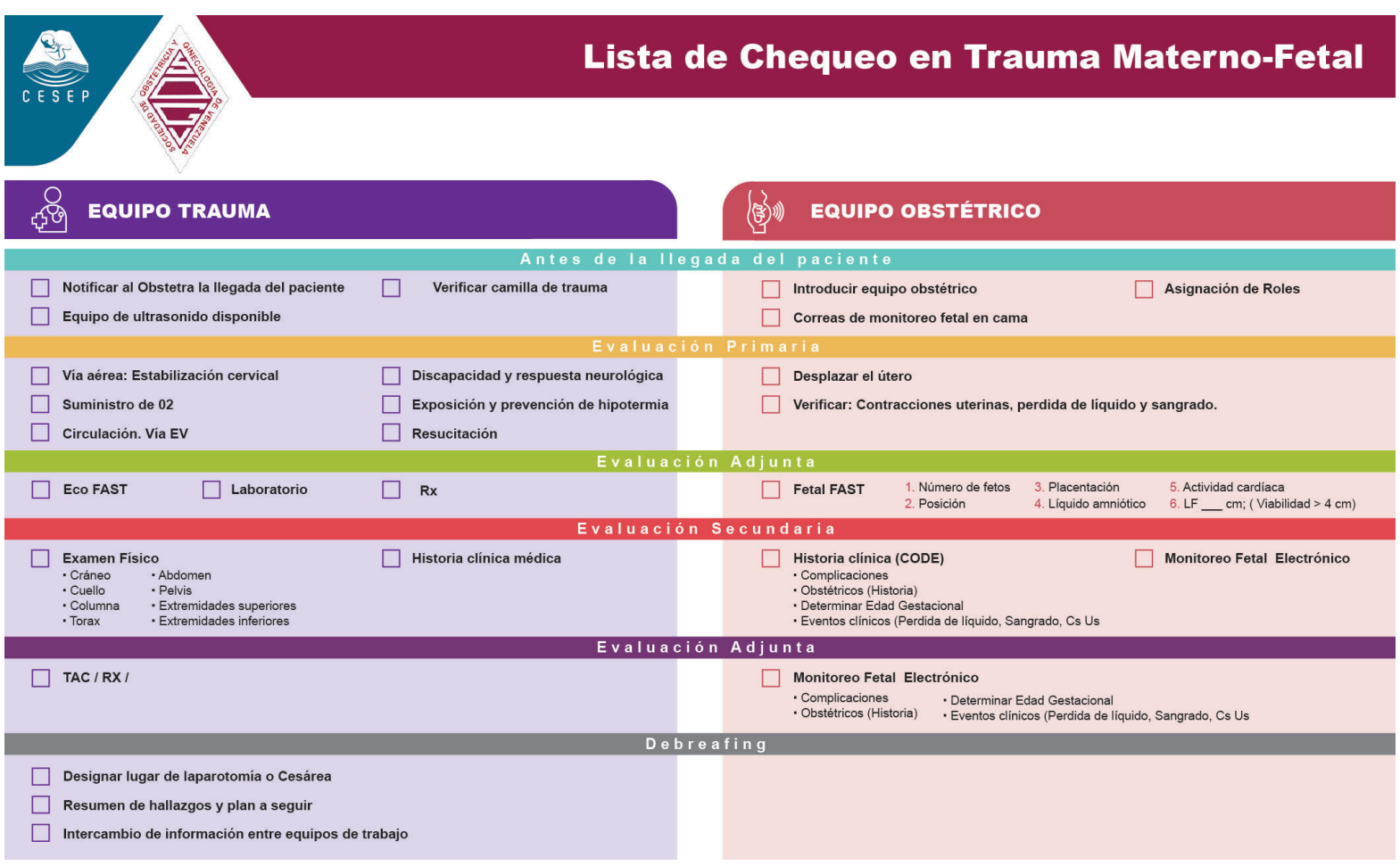

Vol. 80, No 4, diciembre 2020. 


\section{DISCUSIÓN}

Como resultado de esta iniciativa se diseñaron unas listas de chequeo actualizadas según la evidencia actual. Aunque las ayudas cognitivas son una herramienta muy importante en el manejo de eventos críticos, debe existir la conciencia en los líderes de salud acerca de la necesidad de establecer un adecuado programa de implementación de las listas de chequeo.

La evidencia es consistente sobre el efecto favorable de las listas de chequeo en el manejo de pacientes obstétricos críticos y en el desempeño del personal a cargo (32). Resulta complejo asumir que una lista de chequeo se ajuste perfectamente a todas las urgencias que se pueden presentar en una sala de partos (33). En consecuencia, a pesar de la utilidad de las listas de verificación y de otras ayudas cognitivas, un adecuado entrenamientobasado en competencias (conocimientos, habilidades y actitudes) (34), la experiencia clínica y el compromiso con la seguridad de los pacientes siguen siendo los componentes centrales en el manejo de eventos críticos en salas de emergencia. En conclusión, las listas de chequeo, producto de la iniciativa de la Sociedad de Obstetricia y Ginecología de Venezuela quedan a disposición de todo el personal de salud que las requiera para su implementación en escenarios educativos de simulación y en el ejercicio clínico, como una herramienta adicional para la búsqueda de mejores desenlaces en los pacientes que requieren un manejo de alta complejidad en salas de maternidad.

\section{REFERENCIAS}

1. Hirshberg A, Srinivas S. Epidemiology of maternal morbidity and mortality. Semin Perinatol. 2017; 41(6):332-337.

2. Muench M, Canterino J. Trauma in pregnancy. Obstet Gynecol Clin North Am. 2007; 34(3):555-583.

3. Arriaga AF, Bader AM, Wong JM, Lipsitz SR, Berry WR, Ziewacz JE, et al. Simulation-based trial of surgical-crisis checklists. N Engl J Med [Internet]. 2013 [consultado el 16 de mayo 2020]; 368(3):246-
253. Disponible en: https://www.ncbi.nlm.nih.gov/ pubmed/23323901.

4. Ghaferi A, Birkmeyer J, Dimick J. Variation in hospital mortality associated with inpatient surgery. N Engl J Med. 2009; 361(14):1368-1375.

5. Ghaferi A, Birkmeyer J, Dimick J. Complications, failure to rescue, and mortality with major inpatient surgery in medicare patients. Ann Surg. 2009; 250(6):1029-1034.

6. $\mathrm{Hu} \mathrm{X}$, Chen H, Yu M. Exploring the non-technical competencies for on-scene public health responders in chemical, biological, radiological, and nuclear emergencies: a qualitative study. Public Health. 2020; 183:23-29.

7. Goldhaber-Fiebert S, Howard S. Implementing emergency manuals: Can cognitive aids help translate best practices for patient care during acute events? Anesth Analg. 2013; 117(5):1149-1161.

8. Harrison TK, Manser T, Howard SK, Gaba DM. Use of cognitive aids in a simulated anesthetic crisis. Anesth Analg. 2006; 103:551-556.

9. Hepner D, Rubio J, Vasco-Ramírez M, RincónValenzuela D, Ruiz-Villa JO, Amaya Restrepo JC, et al. Listas de chequeo de la Sociedad Colombiana de Anestesiología y Reanimación (S.C.A.R.E.) para el manejo de eventos críticos en salas de cirugía: traducción y actualización basada en la evidencia. Rev Colomb Anest [Internet]. 2017 [consultado el 16 de mayo 2020]; 45 (3):182-199. Disponible en: https:// www.redalyc.org/articulo.oa?id=195152438005

10. Marshall S. The use of cognitive aids during emergencies in anesthesia: A review of the literature. Anesth Analg. 2013;117(5):1162-1171.

11. Palleja Martín, Solari Carlos, Ramírez Mauricio. Listas de chequeo: Crisis en Obstetricia. Anest Analg Reanim [Internet]. 2017 [consultado 16 de mayo de 2020]; 30 (2):13-35. Disponible en: http://www.scielo. edu.uy/scielo.php?script $=$ sci_abstract\&pid $=$ S1688$12732017000300013 \& \operatorname{lng}=\mathrm{es} \& \mathrm{nrm}=$ iso

12. Kohn LT, Corrigan JM, Donaldson MS, editors. To Err is human: Building a safer health system [Internet]. Washington (DC): Institute of Medicine (US) Committee on Quality of Health Care in America. National Academies Press (US); 2000 [consultado 16 de mayo de 2020]. Disponible en: https://www.magonlinelibrary.com/doi/pdf/10.12968/ bjhc.2000.6.9.19311

13. Ziewacz JE, Arriaga AF, Bader AM, Berry WR, Edmondson L, Wong JM, et al. Crisis checklists for 
the operating room: development and pilot testing. J Am Coll Surg [Internet]. 2011 [consultado 16 de mayo de 2020]; 213(2):212-217.e10. Disponible en: https:// pubmed.ncbi.nlm.nih.gov/21658974/

14. Deering S, Rowland J. Obstetric emergency simulation. Semin Perinatol. 2013; 37(3):179-188.

15. Neal J, Hsiung R, Mulroy M, Halpern B, Dragnich A, Slee AE. ASRA checklist improves trainee performance during a simulated episode of local anesthetic systemic toxicity. Reg Anesth Pain Med. 2012; 37(1):8-15.

16. Rueda Fuentes JV, Pinzón Flórez CE, Vasco Ramírez M. Manejo anestésico para operación cesárea urgente: revisión sistemática de la literatura de técnicas anestésicas para cesárea urgente. Rev Colomb Anestesiol. 2012; 40(4):273-86.

17. American College of Obstetricians and Gynecologists' Committee on Patient Safety and Quality Improvement. Committee Opinion No. 680: The use and development of checklists in Obstetrics and Gynecology. Obstet Gynecol [Internet]. 2016 [consultado 16 de mayo de 2020]; 128(5):e237-e240. Disponible en: https:// pubmed.ncbi.nlm.nih.gov/27776075/

18. Main EK, Goffman D, Scavone BM, Low LK, Bingham $\mathrm{D}$, Fontaine PL, et al.; National Partnership for Maternal Safety; Council on Patient Safety in Women's Health Care. National Partnership for Maternal Safety: Consensus Bundle on obstetric hemorrhage. Obstet Gynecol [Internet]. 2015 [consultado 16 de mayo de 2020]; 126(1):155-162. Disponible en: https://pubmed. ncbi.nlm.nih.gov/26241269/

19. Bonet M, Nogueira Pileggi V, Rijken M, Coomarasamy A, Lissauer D, Souza J, et al. Towards a consensus definition of maternal sepsis: results of a systematic review and expert consultation. Reprod Health. 2017; 14(1)67-80.

20. Singer M, Deutschman CS, Seymour CW, Shankar-Hari M, Annane D, Bauer M, et al. The Third International Consensus Definitions for Sepsis and Septic Shock (Sepsis-3). JAMA. 2016; 315(8):801-810.

21. Sutton A, Harper L, Tita A. Hypertensive disorders in pregnancy. Obstet Gynecol Clin North Am. 2018; 45(2):333-347.

22. Altman D, Carroli G, Duley L, Farrell B, Moodley J, Neilson J, et al. Do women with pre-eclampsia, and their babies, benefit from magnesium sulphate? The Magpie Trial: a randomised placebo-controlled trial. Lancet. 2002; 359(9321):1877-1890.

23. Keepanasseril A, Maurya DK, Manikandan K, Suriya J Y, Habeebullah S, Raghavan SS. Prophylactic magnesium sulphate in prevention of eclampsia in women with severe preeclampsia: randomised controlled trial (PIPES trial). J Obstet Gynaecol. 2018; 38(3):305-309.

24. Chandraharan E, Rao S, Belli A, Arulkumaran S. The Triple-P procedure as a conservative surgical alternative to peripartum hysterectomy for placenta percreta. Int $\mathrm{J}$ Gynecol Obstet. 2012; 117(2):191-194.

25. El Gelany S, Mosbeh M, Ibrahim E, Mohammed M, Khalifa E, Abdelhakium A, et al. Placenta Accreta Spectrum (PAS) disorders: incidence, risk factors and outcomes of different management strategies in a tertiary referral hospital in Minia, Egypt: a prospective study. BMC Pregnancy Childbirth. 2019; 19(1):313320.

26. Palacios-Jaraquemada JM, Fiorillo Á, von Petery F, Colaci D, Leguizamón G. Uterine repair and successful pregnancy after myometrial and placental rupture with massive haemoperitoneum. BJOG. 2009; 116(3):456460.

27. Jeejeebhoy FM, Zelop CM, Lipman S, Carvalho B, Joglar J, Mhyre JM, et al. Cardiac arrest in pregnancy: a scientific statement from the American Heart Association. Circulation. 2015; 132(18):1747-1773.

28. Benson M, Padovano A, Bourjeily G, Zhou Y. Maternal collapse: Challenging the four-minute rule. E Bio Medicine [Internet]. 2016 [consultado 16 de mayo de 2020]; 6: 253-257. Disponible en: https://www.ncbi. nlm.nih.gov/pmc/articles/PMC4856753/

29. Adams J, Cepeda Brito JR, Baker L, Hughes PG, Gothard MD, McCarroll ML, et al. Management of maternal cardiac arrest in the third trimester of pregnancy: A simulation-based pilot study. Crit Care Res Pract [Internet]. 2016 [consultado 16 de mayo de 2020]; 2016: 283765. Disponible en: https://pubmed. ncbi.nlm.nih.gov/27555967/

30. Neumar RW, Shuster M, Callaway CW, Gent LM, Atkins DL, Bhanji F, et al. Part 1: Executive Summary: 2015 American Heart Association Guidelines Update for Cardiopulmonary Resuscitation and Emergency Cardiovascular Care. Circulation. 2015; 132(18 Suppl 2):S315-367.

31. Link MS, Berkow LC, Kudenchuk PJ, Halperin HR, Hess EP, Moitra VK, et al. Part 7: Adult advanced cardiovascular life support: 2015 American Heart Association Guidelines Update for Cardiopulmonary Resuscitation and Emergency Cardiovascular Care. Circulation. 2015; 132 (18 Suppl 2):444-464.

32. Society for Maternal-Fetal Medicine (SMFM); 
Bernstein PS, Combs CA, Shields LE, Clark SL, Eppes CS; SMFM Patient Safety and Quality Committee. The development and implementation of checklists in obstetrics. Am J Obstet Gynecol [Internet]. 2017 [consultado 16 de mayo de 2020]; 217(2):B2B6. Disponible en: https://www.ajog.org/action/ showPdf?pii=S0002-9378\%2817\%2930643-9

33. Borshoff D. The limitations of crisis checklists. Anesth Analg. 2014; 118(6):1387-1388.
34. Kain ZN, Fitch JC, Kirsch JR, Mets B, Pearl RG. Future of anesthesiology is perioperative medicine: a call for action. Anesthesiology. 2015; 122(6):1192-1195.

Recibido: 22 de mayo de 2020

Aprobado: 10 de julio de 2020 